\title{
Thin Gap Gas Chambers for the DELPHI Endcaps
}

\author{
E. Albrecht, O. Ullaland \\ CERN, Geneva, Switzerland \\ M. Bozzo, F. Fontanelli, V. Gracco, E. Mongiardini, A. Morelli, P. Morettini, \\ A. Petrolini, G. Piana \\ INFN and Università di Genova, Via Dodecaneso 33, I-16146 Genova, Italia \\ W. Adam, J. Hrubec \\ HEPHY Institut für Hochenergiephysik, Wien, Österreich
}

\begin{abstract}
Thin Gap Gas Chambers have been proposed for an upgrade of the endcaps of the DELPHI detector at LEP.

Two full size chambers have been built and a study of the optimal operating conditions has been carried out. In this paper the main construction parameters are discussed and test results are given. Tests of the electronic readout were performed and the general feasibility of the detector is demonstrated.
\end{abstract}

Presented by G.Piana at the IEEE-NSS96 Conference, Anaheim, California, USA,

November 1996.

Submitted to IEEE Transactions on Nuclear Science. 


\title{
Thin Gap Gas Chambers for the DELPHI Endcaps
}

\author{
W. Adam ${ }^{3}$, E. Albrecht ${ }^{1}$, M. Bozzo ${ }^{2}$, F. Fontanelli ${ }^{2}$, V. Gracco ${ }^{2}$, \\ J. Hrubec ${ }^{3}$, E. Mongiardini ${ }^{2}$, A. Morelli ${ }^{2}$, P. Morettini ${ }^{2}$, A. Petrolini' ${ }^{2}$, \\ G. Piana ${ }^{2}$ and O. Ullaland ${ }^{1}$ \\ ${ }^{1}$ CERN, Geneva, Switzerland \\ ${ }^{2}$ INFN and Università di Genova, Via Dodecaneso 33, I-16146 Genova, Italia \\ ${ }^{3} \mathrm{HEPHY}$ Institut für Hochenergiephysik, Wien, Österreich
}

\begin{abstract}
Thin Gap Gas Chambers have been proposed for an upgrade of the endcaps of the DELPHI detector at LEP.

Two full size chambers have been built and a study of the optimal operating conditions has been carried out. In this paper the main construction parameters are discussed and test results are given. Tests of the electronic readout were performed and the general feasibility of the detector is demonstrated.
\end{abstract}

\section{INTRODUCTION}

The recent DELPHI [1] improvements in the forward regions [2] raised the possibility of improving the trigger and tracking performances at low polar angle with a further high efficiency detecting plane.

The detector must fit with the present DELPHI trigger structure and allow for fast signal treatment. The very narrow free space available in the endcap region and the necessity to build a large detector with short radiation length made the Thin Gap Gas Chamber [3] a good option.

The chamber structure is made of multilayers printed circuit boards (PCB). The trigger geometry requirement was met by a high granularity pattern of triangular pads on one of the two cathode planes. The other cathode plane is etched with strips either radial or transverse and gives a further point for tracking at a large distance $(\mathrm{z}= \pm 167 \mathrm{~cm})$ from the DELPHI interaction point. The same space and trigger requirements also constrained the electronics design. More than 500 channels per chamber are discriminated and elaborated by programmable processors on printed boards placed on top of the chambers. An optical link is foreseen to take the processed information out of the detector to the DELPHI data acquisition system.

Thin multiwire chambers operating in a high gain mode were first developed in 1983 [3] and then successfully used by the OPAL experiment at the Large Electron Positron collider (LEP) at CERN as a large scale detector for the pole tip hadron calorimeter and endcap presampler [4]. Due to the good timing information [5] Thin Gap Gas Chambers (TGGC) are also proposed for the first level muon trigger system of the ATLAS experiment at the Large Hadron Collider (LHC) [6]. The chamber works in near saturated gas amplification mode with a highly quenching gas mixture. The chamber signals are fast $(\leq 20 \mathrm{~ns})$ and large $(\sim 1 \mathrm{pC})$ and can therefore be directly discriminated without pre-amplification.

With respect to previous applications this work proves the feasibility of this kind of detector when a high granularity is required on a large size detector and therefore many readout channels as well as VLSI electronics are involved. 


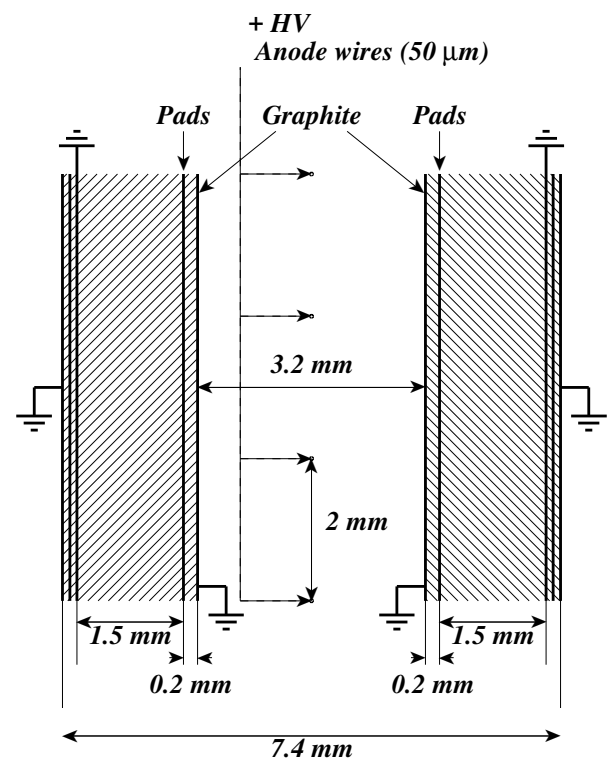

Fig. 1 Schematic drawing of the TGGC

Two full size chambers have been tested in the CERN SPS beam test area, the parameters of the chamber were optimized to give stable operation conditions. Direct tests of both the chamber and the readout electronics proved the general feasibility of the detector.

\section{Thin GAP GAS CHAMBER}

The schematic cross-section of the Thin Gap Gas Chamber is shown in figure 1. A plane of $50 \mu \mathrm{m}$ diameter wires with $2 \mathrm{~mm}$ spacing is placed between two cathode planes, made of multilayers PCBs and separated by a $3.2 \mathrm{~mm}$ gap. The surface facing the wire plane is made out of $200 \mu \mathrm{m}$ glass fiber reinforced epoxy (FR4) and is covered with graphite [7]. This graphite layer has a typical surface resistance of $\sim 5 \mathrm{M} \Omega$ /square. The signal induced on the resistive cathodes is picked-up by the underlying copper pads or strips. Transmission lines between pads or strips and the electronics are made with the external PCB layers. The total thickness of the chamber is $7.4 \mathrm{~mm}$. Besides being a compact and reliable structure for the connections, this solution has the further advantage of using standard industrial multilayers PCB techniques. The chambers are usually operated with a mixture of $\mathrm{CO}_{2}$ :n-pentane 55:45. Applying a typical voltage between 3.5 and $3.7 \mathrm{kV}$ the operation is in a nearly saturated mode with a gas amplification of the order of $\sim 10^{7}$.

\section{OVERVIEW OF THE DETECTOR AND DESIGN CONSIDERATIONS}

The present forward track trigger of the DELPHI experiment is based on the wire information from the Time Projection Chamber (TPC) and the two drift chambers A and $\mathrm{B}$ (FCA and FCB) [8]. The angular coverage of the TPC starts at about $17^{\circ}$ and below this angle the trigger only relies on the two Forward Chambers. The detector that is proposed to improve the trigger and tracking performances covers the polar angle region between $10^{\circ}$ and $30^{\circ}$ and is planned to be placed just after the present Forward Chamber A.

Each endcap can be instrumented with two sets of six $60^{\circ}$ trapezoidal chambers. The two sets fit the $\sim 1.5 \mathrm{~cm}$ gap between the present FCA and the Forward Ring Imaging Cherenkov. The planes will be staggered to improve the geometrical acceptance.

Since the proposed detector has to follow the DELPHI trigger structure each plane of chambers has to provide three coordinates to be realized with a single cathode plane. This is obtained by etching the cathode plane in triangular pads as shown in Fig 2 and properly 
combining the pads rows. Below about $17^{\circ}$ the small pads have a surface of $5.68 \mathrm{~cm}^{2}$ while above this angle the chamber granularity may be safely reduced using bigger triangular pads $\left(22.7 \mathrm{~cm}^{2}\right)$.

Only one of the two cathode planes of the TGGC is shaped with triangular pads. The other cathode plane is etched with either radial or transverse strips which give a $\phi$ and $\theta$ information on the track. Each strip is placed at a defined angle and the pitch between two strips is $\delta \phi=0.22^{\circ}$ or $\delta \theta=0.1^{\circ}$.

A further point for the tracking can therefore be given with high efficiency. This point is useful for solving the left-right ambiguities in the drift chambers.

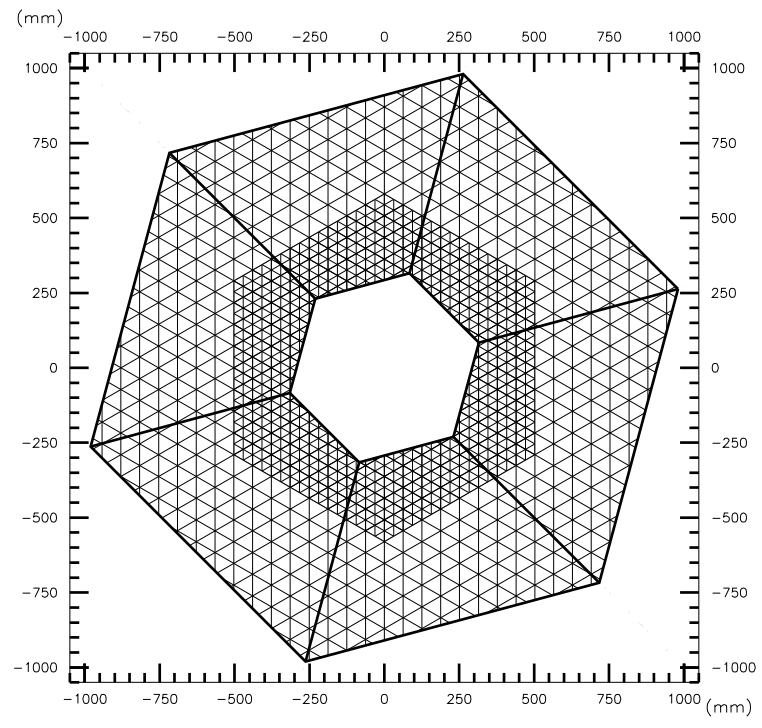

Fig. 2 One plane of six TGGC chambers. Two staggered planes fit in the space available in each endcap of the DELPHI detector. The structure of the pads is visible.

\section{THE CHAMBER CONSTRUCTION}

As previously described the two half shells composing the chamber are industrially produced PCBs [12].

The PCB surface is then cleaned with alcohol and a graphite paint is sprayed and polished. A $10 \mathrm{~mm}$ wide ceramic circuit board [11] runs along the long sides of the chambers. This board incorporates the high voltage bus, the decoupling resistor to each wire and the wire connection. The resistor is printed on the ceramic support $(470 \mathrm{k} \Omega)$. The wire is connected and held in place with epoxy.

Two types of $50 \mu \mathrm{m}$ diameter wires have been tried, gold-plated tungsten [9] and stainless steel [10] strung at a mechanical tension of $300 \mathrm{~g}$. No operational difference between the two types have been observed. To avoid wire sagging and improve the chamber rigidity spacers are placed about $20 \mathrm{~cm}$ apart. The chamber is then closed with epoxy to get a compact and stiff structure.

The electronics for both strips and pads is placed in two independent multilayer PCBs connected to the upper part of the chamber.

The 273 pads and 266 strips channels are discriminated and digitally processed to produce prompt trigger information which is then transmitted through the serial optical link. The functional scheme is shown in Fig 3. 


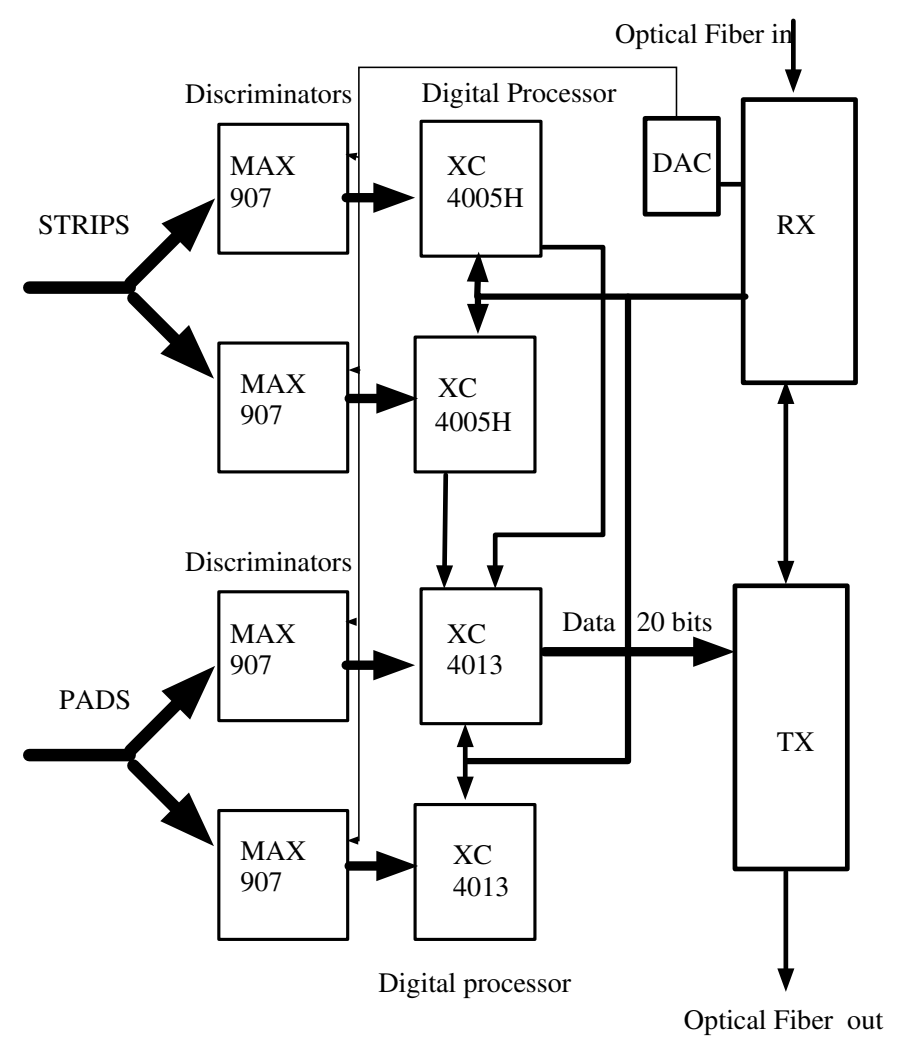

Fig. 3 Electronics functional scheme

\section{ELECTRONics}

Also in the electronics PCB particular care has been devoted to avoid electromagnetic interference between channels and from external noise collection.

The discriminators which have been used [13] have $5 \mathrm{mV}$ built-in hysteresis which strongly suppresses any auto-oscillations or spurious switching. They have also been chosen for their very low power consumption $(3.5 \mathrm{~mW}$ per channel) to limit the heat dissipation. The response time is not a critical parameter for the DELPHI trigger therefore the discriminators $40 \mathrm{~ns}$ propagation delay is well suited for our application. A $1 \mathrm{k} \Omega$ input impedance has been chosen for the discriminator input. Different thresholds for pads and strips channels can be remotely set by means of a serially programmable DAC.

Discriminated signals from pads are processed by programmable circuits [14] to compute the $(\mathrm{x}, \mathrm{u}, \mathrm{v})$ coordinate which are then immediately sent to the first level DELPHI forward trigger processor. An optical link is foreseen between the detector and the counting room. This choice is mainly due to the severe space limitations, but it also avoids adding unnecessary material into the detector volume and has the advantage of being immune to electromagnetic pick-up. The transmission is carried out by means of an optical transceiver chosen for high transmission rate and low cost [15]. It also contains a low power standby mode which enables to reduce the power consumption when no communication is needed.

With a clock frequency of the programmable processor set to $20 \mathrm{MHz}$, the trigger information (58 coordinates) is sent in $250 \mathrm{~ns}$. In case of positive first level trigger the full information for pads and strips is sent in about $2.5 \mu \mathrm{s}$. 


\section{TESTS}

\section{A. The Experimental Setup}

To study the behaviour of the chamber under different operating conditions a series of tests have been carried out using a $20 \mathrm{GeV} / \mathrm{c}$ pion beam at the CERN SPS. The trigger was realized with two scintillation counters and the signals from pads and strips digitized by an ADC.

\section{B. Efficiency and Charge Measurement}

Due to the short drift time the TGGC have a narrow timing spread and most of the hits have been observed inside a $\sim 20 \mathrm{~ns}$ time window. A gate width of $65 \mathrm{~ns}$ has been used for the ADC to fully contain the signals. With the described setup the average collected charge as a function of the anode voltage has been measured for the different cathodes shapes as shown in figure 4 . The pedestal is gaussian with a $\sigma$ corresponding to $0.08 \mathrm{pC}$.

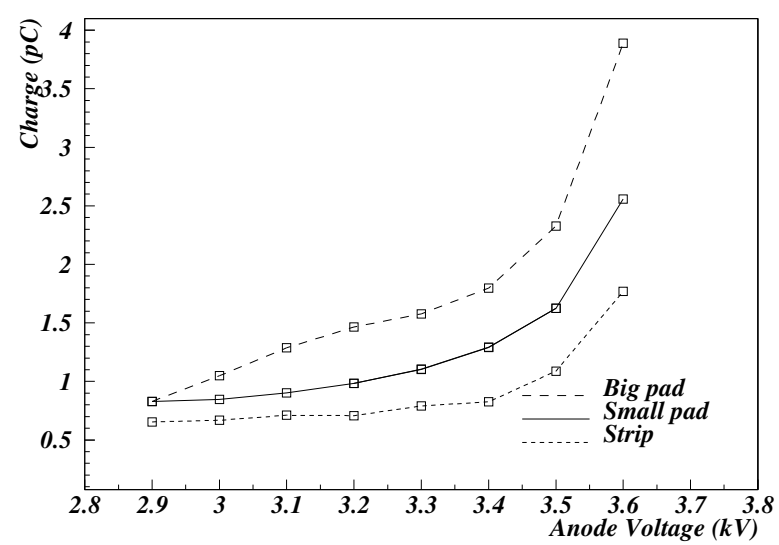

Fig. 4 Average charge collected by the cathodes ( triangular pads and strip ) for the gas mixture (a). Small and big pads are defined in Sec. III.

The chamber is therefore insensitive to pick-up noise. Events with a signal $2.5 \sigma$ above the pedestal are used to evaluate the efficiency. The signal itself is fitted and the distance between the pedestal and the signal peaks define the separation shown in figure 5 . The effect

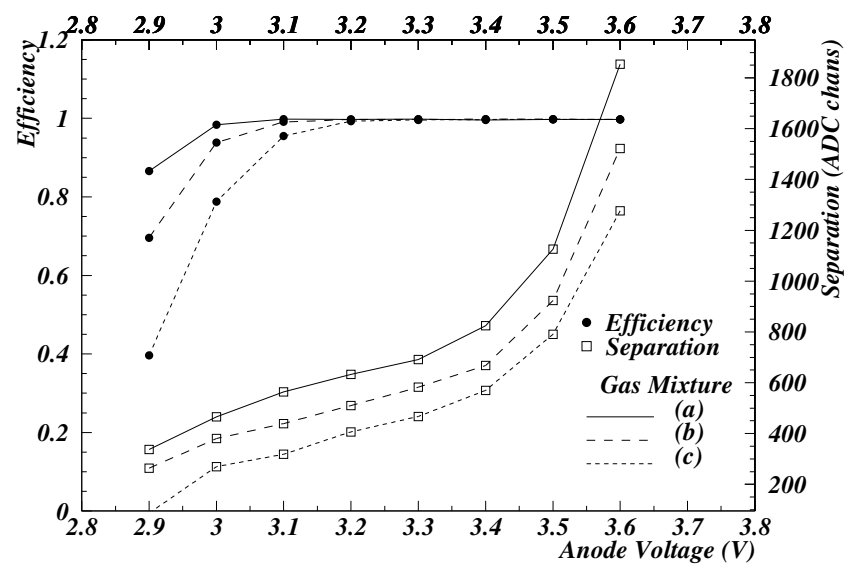

Fig. 5 Efficiency and separation for different gas mixtures (big pad).

of the gas composition on the signals has been studied as a function of the anode voltage, see Fig 5. Different gas mixtures $\mathrm{CO}_{2}$ n-pentane have been used as in table 1. The gas mixture (c) is a standard one (48:52) for the TGGC while the other two have considerably 
Table 1

Gas mixtures $\mathrm{CO}_{2}$ :n-pentane relative percentages used in the test

\begin{tabular}{|c|c|}
\hline $\begin{array}{c}\mathrm{Gas} \\
\text { mixture }\end{array}$ & $\begin{array}{c}\mathrm{CO}_{2} \text { :n-pentane } \\
\text { ratio }\end{array}$ \\
\hline $\mathrm{a}$ & $68: 32$ \\
$\mathrm{~b}$ & $61: 39$ \\
$\mathrm{c}$ & $48: 52$ \\
\hline
\end{tabular}

lower concentrations of n-pentane. As shown in figure 5 bigger signals have been observed the lower the concentration of n-pentane. Also the efficiency plateau is reached at a lower anode voltage. No change in the stability of the chamber was observed while decreasing the quencher percentage well below the traditionally used amount. When the beam is centered on a small pad $1.5 \%$ of events give additional signal on one of the surrounding pads. This is the upper limit to the cross-talk.

\section{Tests of the Front-End Electronics}

With a discriminator threshold of $4.2 \mathrm{mV}$ the efficiency plateau is reached at about $3.3 \mathrm{kV}$. The built-in hysteresis in the discriminator prevents any auto-oscillation and highly reduce random triggers from noise. The global timing jitter was observed to be within $\sim 20 \mathrm{~ns}$ for most of the signals. The programmable circuit which perform the trigger coordinate computation acts according to the specifications.

\section{SUMMARY}

A detector based on Thin Gap Gas Chambers has been proposed for the use in the DELPHI Endcap. With respect to previous applications this work demonstrates the feasibility of this kind of detector when a high granularity is required on a large size detector.

Measurements on full prototypes have been carried out and a gas with a very low npentane content is shown to allow for an efficiency greater than $99 \%$ at a voltage $\geq 3.1 \mathrm{kV}$. The total charge is measured at $3.5 \mathrm{kV}$ between $0.8 \mathrm{pC}$ and $2.4 \mathrm{pC}$ depending on the cathode size, which is a factor 10-30 above the pedestal.

The cross-talk between channels is observed to be less than $1.5 \%$. The front-end electronics have been tested on the chamber signals and found to meet the design specifications. The tests carried out on the complete prototypes have shown the general feasibility of the detector.

\section{ACKNOWLEDGMENTS}

We would like to thank G. Mikenberg for his advise and the Weizmann Institute of Science for the help given with the chamber graphite layer; G. Leder and M. Pernicka of the HEPHY Institut für Hochenergiephysik for many useful discussions and suggestions, P. Adzic and A. Markou. of the N.C.S.R. Demokritos and I. van Vulpen of Nikhef for the help given with the tests, A. Bevilacqua, P. Musico and F. Siccardi for the CAD work. We express our gratitude to L. Gatignon and collaborators for their help in the set-up of the beam line.

We wish also to thank J.E. Augustin, H. Foeth and D. Treille for initiating this work.

\section{REFERENCES}

[1] P. Aarnio et al. , The DELPHI detector at LEP , Nucl. Instr. and Meth. A303, (1991) 233.

[2] V. Chabaud et al. , The DELPHI silicon strip microvertex detector with double sided, Nucl. Instrum. Meth. A368(1996), 314.

K. H. Becks et al. , Progress in the construction of the DELPHI Pixel Detector, III Int. Work. on Semiconductor Pixel Detectors for Particles and X-Ray (1996), Bari (Italy) 
[3] S. Majewski et al., A thin multiwire chamber operating in the high multiplication mode, Nucl. Instr. and Meth. , 217 (1983) 265.

[4] G. Bella et al., Development of calorimeters using thin chambers operating in high gain mode, Nucl. Instr. and Meth., A252 (1986) 503.

S. Dado et al. , A new high gain thin gap detector for the OPAL hadron calorimeter, Nucl. Instr. and Meth. , A252 (1986) 511. G. Mikenberg, Thin-gap chambers for hadronic calorimetry, Nucl. Instr. and Meth. , A265 (1988) 223.

G. Beard et al., Thin, high gain wire chambers for electromagnetic presampling in OPAL, Nucl. Instr. and Meth., A286 (1990) 117.

[5] Y. Arai et al. , Timing Optimization of Thin Gap Chambers for the Use in the ATLAS Muon Endcap Trigger, Nucl. Instrum. Meth. A367(1995), 398.

[6] ATLAS Collaboration, Technical Proposal CERN/LHCC/94-43, (1994), 116.

[7] Graphite 33 and Plastik 70, Kontakt-Chemie (Germany)

[8] V. Bocci et al., Architecture and performance of the DELPHI trigger system, CERN-PPE 94-141. P. Aarnio et al. (DELPHI Collaboration), Nucl. Instr. and Meth., A303 (1991) 233.

[9] 50 $\mathrm{m}$ gold-plated tungsten wire, Luma Metall, Kalmar, (Sweden)

[10] 50 $\mu \mathrm{m}$ stainless steel wire, Microfil Industries SA, Renens, (Switzerland)

[11] Custom Ceramic Board, NEOHM Componenti s.r.l., Torino (ITALY)

[12] Custom multilayer Printed Circuit Board, DITRON s.r.l., Novara (ITALY)

[13] MAX 907 Dual High Speed, Ultra Low Power TTL Comparators, Maxim Integrated Products (USA)

[14] XC4005 and XC4013 PCI, Xilinx Inc, (USA)

[15] FTR-8510 Low Cost Gigabit Optical Transceiver, Finisar Corporation (USA) 